\title{
To Predict the Requirement of Pharmacotherapy by OGTT Glucose Levels in Women with GDM Classified by the IADPSG Criteria
}

\author{
Gülen Yerlikaya, ${ }^{1}$ Veronica Falcone, ${ }^{1}$ Tina Stopp, ${ }^{1}$ Martina Mittlböck, ${ }^{2}$ Andrea Tura (D), ${ }^{3}$ \\ Peter Husslein, ${ }^{1}$ Wolfgang Eppel, ${ }^{1}$ and Christian S. Göbl $\mathbb{1}^{1}$ \\ ${ }^{1}$ Department of Obstetrics and Gynecology, Division of Obstetrics and Feto-Naternal Medicine, Medical University of Vienna, \\ Vienna, Austria \\ ${ }^{2}$ Center of Medical Statistics, Informatics, and Intelligent Systems, Section for Clinical Biometrics, Medical University of Vienna, \\ Vienna, Austria \\ ${ }^{3}$ Metabolic Unit, Institute of Neuroscience, National Research Council, Padova, Italy
}

Correspondence should be addressed to Christian S. Göbl; christian.goebl@meduniwien.ac.at

Received 30 January 2018; Revised 31 March 2018; Accepted 5 April 2018; Published 8 May 2018

Academic Editor: Kazuya Yamagata

Copyright ( 2018 Gülen Yerlikaya et al. This is an open access article distributed under the Creative Commons Attribution License, which permits unrestricted use, distribution, and reproduction in any medium, provided the original work is properly cited.

The aim of this study was to assess the association between OGTT glucose levels and requirement of pharmacotherapy in GDM patients classified by the IADPSG criteria. This study included 203 GDM patients (108 managed with lifestyle modification and 95 requiring pharmacotherapy). Clinical risk factors and OGTT glucose concentrations at 0 (G0), 60 (G60), and 120 min (G120) were collected. OGTT glucose levels were significantly associated with the later requirement of pharmacotherapy (ROC-AUC: 71.1, 95\% CI: 63.8-78.3). Also, the combination of clinical risk factors (age, BMI, parity, and pharmacotherapy in previous gestation) showed an acceptable predictive accuracy (ROC-AUC: 72.1, 95\% CI: 65.0-79.2), which was further improved when glycemic parameters were added (ROC-AUC: 77.5, 95\% CI: 71.5-83.9). Random forest analysis revealed the highest variable importance for G0, G60, and age. OGTT glucose measures in addition to clinical risk factors showed promising properties for risk stratification in GDM patients classified by the recently established IADPSG criteria.

\section{Introduction}

The International Associations for Diabetes in Pregnancy Study Groups (IADPSG) guidelines for gestational diabetes mellitus (GDM) classification recommends a primary testing until the early third trimester by a $2 \mathrm{~h}-75 \mathrm{~g}$ oral glucose tolerance test (OGTT) $[1,2]$. However, it was found that these recommendations resulted in a markedly increased number of GDM cases [3] containing a heterogenic group of pregnant women on the wide range of disturbed glucose metabolism. Therefore, most patients achieve adequate glycemic control with lifestyle modification while others need intensified pharmacologic therapy.

An early stratification into low- and high-risk patients could markedly improve the efficiency of treatment. Within the last years, different risk factors were identified which are associated with the requirement of glucose-lowering medications in women with GDM. These include age, prepregnancy BMI, history of GDM in previous pregnancy, and others [4-7]. Although previous studies indicated that increased OGTT glucose concentrations were related to adverse gestational and fetal outcome $[8,9]$, their specific performance for the prediction of pharmacotherapy in patients meeting the IADPSG criteria is less well investigated.

Therefore, this study aims to examine the ability of OGTT glucose levels to distinguish between low- (achieving glycemic targets with lifestyle modification) and high-risk (requiring pharmacotherapy) GDM patients diagnosed by the IADPSG criteria. Moreover, their contribution to discriminate low- and high-risk patients additional to more common and routinely documented risk factors should be assessed. 


\section{Materials and Methods}

In this retrospective case-control study, we included 203 pregnant women diagnosed with GDM (108 managed with lifestyle modification and 95 requiring pharmacotherapy with insulin $(n=80)$, metformin $(n=11)$, or both $(n=4))$, who attended our outpatient clinic between May 2015 and January 2017. GDM was diagnosed via universal testing by a 75 g-OGTT at 24-28 weeks of gestation, according to the IADPSG recommendations $[1,2]$. Intensified lifestyle modification (ILS) including medical nutrition therapy was used as the first line intervention. All patients were instructed for capillary blood glucose monitoring and informed about glycemic treatment targets. Follow-ups in two to three weeks' time were scheduled, and blood glucose levels were reviewed during each appointment. The pharmacologic intervention was started at any time point when blood glucose targets were not achieved (i.e., $<95 \mathrm{mg} / \mathrm{dl}$ at fasting or $<140 \mathrm{mg} / \mathrm{dl}$ one hour after each meal) according to the current guidelines [10]. The following variables were collected from the medical records: age,gravidity, parity, prepregnancy BMI, history of GDM, first-degree relative with diabetes, and OGTT results at $0 \mathrm{~min}$ (G0), $60 \mathrm{~min}$ (G60), and $120 \mathrm{~min}$ (G120). Patients with preexisting diabetes as well as multiple pregnancies or those with missing OGTT glucose values were excluded. The study was approved by the Ethics Committee of the Medical University of Vienna and performed in accordance with the Declaration of Helsinki.

2.1. Statistical Analysis. Continuous and categorical variables were summarized as the means \pm SD or median (IQR) and counts and percentages and compared by Student's $t$-test (or the Wilcoxon rank sum test for skewed distributed variables) and Pearson's chi-square test. Binary logistic regression was used to assess the probability of initiation of pharmacotherapy by OGTT glucose levels and risk factors such as age, prepregnancy BMI, parity, and history of GDM and others. Thereby, the predictive accuracy of the models was expressed as the area under the receiver operating characteristic curves (ROC-AUC). Unbiased recursive partitioning was performed, whereby decision trees were created by the conditional inference (Ctree) framework [11]. Thereby, random decision forests with ntree $=5000$ were created to derive measures of variable importance (i.e., the average difference in predictive accuracy before and after permutation of a predictor variable over all 5000 trees). As supporting time-to-event analysis, the competing risk model of Fine and Gray [12] was conducted where preterm delivery (before $38+0$ weeks of gestation) is considered a competing event to the start of pharmacotherapy. As single observations were missing at random for some covariables (G0: 1.5\%; G60: 7.4\%; G120: $8.4 \%$; BMIPK: $1.0 \%$; and history of GDM: $10.8 \%$ ), multivariate imputations by chained equations were performed to estimate missing observations by the median value of 50 complete data sets. These imputations were used in logistic and proportional hazard regression models in order to achieve a maximum of information from our data. Group-specific comparisons of raw data are provided in Table 1. Statistical analysis was performed with R (version 3.4.1) and contributed packages ("mice" for data imputations as well as "pROC," "party," and "cmprsk" for data analysis) [13]. A two-sided $p$ value $\leq 0.05$ was considered statistically significant. $p$ values were interpreted in an explorative manner aiming to generate new hypotheses, and no adjustment for multiple statistical testing was used if not otherwise indicated.

\section{Results}

A descriptive comparison of the data is provided in Table 1. It was found that mothers requiring pharmacotherapy were older, had higher BMI, and reached higher plasma glucose concentrations during the OGTT. Although univariable analysis revealed significant associations between plasma glucose levels and risk for pharmacologic intervention, the relative contribution of each glucose measure including their mean value was only modest to fair (ROC-AUC ranged between 60.2 and $65.6 \%$ as provided in Figure 1(a)-1(c)). The predictive performance was, however, markedly improved when the information of all three OGTT glucose measurements was combined by multiple logistic regression (model 1: ROC-AUC: 71.1, Figure $1(\mathrm{~d})$ ). Also, the combination of clinical variables (age, pregestational BMI, parity, history of GDM in previous gestation, family history with type 2 diabetes, and time of diagnosis) showed an acceptable discrimination between low- and high-risk patients (model 2: ROCAUC: 72.1 , Figure $1(\mathrm{e}) ; p=0.834$ versus model 1 ), which was further improved when OGTT glucose levels were included as additional predictors (model 3: ROC-AUC: 77.5, Figure 1 (f); $p=0.030$ versus model 1 and $p=0.033$ versus model 2). Random forest analysis revealed the highest variable importance for G0 $\left(1.11 \times 10^{-2}\right)$, G60 $\left(0.96 \times 10^{-2}\right)$, and age $\left(0.80 \times 10^{-2}\right)$, while the importance of the remaining predictors was shown to be inferior (G120: $0.24 \times 10^{-2}$, BMI: $0.40 \times 10^{-2}$, parity: $0.05 \times 10^{-2}$, history of GDM: $0.31 \times 10^{-2}$, family history with type 2 diabetes: $-0.17 \times 10^{-2}$, and time of diagnosis: $0.13 \times 10^{-2}$ ). An exemplary decision tree with possible cut-off values for G0, G60, and age is also provided. A very low risk for pharmacotherapy was observed for patients with $\mathrm{G} 0 \leq 81 \mathrm{mg} / \mathrm{dl}$, whereas patients with G0>81 and G60>212 had a very high risk for pharmacologic intervention (Figure 2). Pharmacotherapy was initiated at a median time of 31.1 weeks of gestation. G0 (HR: 1.12 , 95\% CI: $1.01-1.24$, for an increase of $10 \mathrm{mg} / \mathrm{dl}$ ), G60 (HR: 1.14, 95\% CI: 1.05-1.24, for an increase of $10 \mathrm{mg} /$ $\mathrm{dl}$ ), and age (HR: 1.25, 95\% CI: 1.04-1.51, for an increase of 5 years) were also found to be independent predictors for pharmacologic intervention by time-to-event analysis, accounting for preterm delivery (before $38+0$ weeks). Moreover, the combined information of G0, G60, and age showed a fair discrimination when included in a logistic regression model to predict the probability for delivering LGA offspring (ROC-AUC: 64.7\%, 95\% CI: 54.1-75.3). However, only G0 reached a significance in this model $(p=0.012)$. 
TABLE 1: Characteristics of the study population.

\begin{tabular}{|c|c|c|c|c|c|}
\hline & $n$ & GDM-LSI $(n=108)$ & $n$ & GDM-PT $(n=95)$ & $p$ value \\
\hline Age (years) & 108 & $31.5 \pm 5.9$ & 95 & $34.6 \pm 5.9$ & $<0.001$ \\
\hline BMI $\left(\mathrm{kg} / \mathrm{m}^{2}\right)$ & 107 & $27.8 \pm 6.4$ & 94 & $30.1 \pm 6.9$ & 0.014 \\
\hline Fertility treatment & 108 & $6(5.6 \%)$ & 95 & $8(8.4 \%)$ & 0.421 \\
\hline Gravidity (count) & 108 & $3.0(2.0-4.0)$ & 95 & $3.0(2.0-5.0)$ & 0.029 \\
\hline Gravidity $(>1)$ & 108 & $87(80.6 \%)$ & 95 & $83(87.4 \%)$ & 0.189 \\
\hline Parity (count) & 108 & $1.0(0.0-2.0)$ & 95 & $1.0(1.0-2.5)$ & 0.054 \\
\hline Parity $(\geq 1)$ & 108 & $73(67.6 \%)$ & 95 & $74(77.9 \%)$ & 0.101 \\
\hline pGDM & 102 & $19(18.6 \%)$ & 87 & $34(39.0 \%)$ & 0.002 \\
\hline G0 (mg/dl) & 105 & $90.0 \pm 10.8$ & 95 & $98.8 \pm 19.2$ & $<0.001$ \\
\hline G60 (mg/dl) & 101 & $173.2 \pm 29.5$ & 87 & $189.0 \pm 30.8$ & $<0.001$ \\
\hline G120 (mg/dl) & 98 & $129.7 \pm 29.0$ & 88 & $140.4 \pm 33.9$ & 0.021 \\
\hline Gmean (mg/dl) & 98 & $130.6 \pm 15.2$ & 87 & $141.7 \pm 20.3$ & $<0.001$ \\
\hline Week diagnosis & 108 & $28.0(26.4-30.3)$ & 95 & $27.7(25.6-29.4)$ & 0.032 \\
\hline Week treatment start & & - & 95 & $31.1(28.0-33.3)$ & \\
\hline $\mathrm{BW}(\mathrm{pct})$ & 108 & $50.5(24.0-70.5)$ & 95 & $50.0(26.0-86.0)$ & 0.427 \\
\hline LGA & 108 & $12(11.1 \%)$ & 95 & $21(22.1 \%)$ & 0.034 \\
\hline
\end{tabular}

Data are mean \pm standard deviations or median and interquartile range (IQR) for pregnant women affected by GDM and treated with lifestyle modification (GDM-LSI) or requiring pharmacologic treatment (GDM-PT). BMI: pregestational body mass index; pGDM: previous pregnancy with gestational diabetes mellitus; G0: fasting plasma glucose; G60: plasma glucose 60 min after oral glucose load; G120: plasma glucose 120 min after oral glucose load; BW: birth weight; LGA: large for gestational age offspring.

\section{Discussion}

This study assessed the association between glucose measures derived from a diagnostic $75 \mathrm{~g}$-OGTT and future requirement of pharmacotherapy in women classified with GDM according to the IADPSG recommendations. Albeit the individual glucose measurements (G0, G60, and G120) were significantly associated with the risk of requiring glucoselowering medication, their predictive performance was markedly improved when the information of all three OGTT glucose values was combined by multivariable logistic regression. The model discrimination of clinical risk factors was also improved when OGTT glucose measurements were added as further predictors. Thereby, G0 followed by G60 and age achieved the highest variable importance measures.

These findings are of clinical relevance: in 2010, the IADPSG recommended novel criteria for GDM classification based on one-step OGTT testing in all pregnant women [1]. These criteria were more recently adopted by the WHO and several local health care organizations [2]. However, it was criticized that this novel established strategy would substantially increase the number of GDM patients by additionally identifying very subtle alterations in disturbed glucose metabolism [14]. This limitation of the IADPSG approach emphasizes the need for accurate risk stratification early after diagnosis to separate low- from high-risk pregnancies, that is, patients who achieve adequate glycemic control with lifestyle modification and those who require more intensified treatment strategies. Although already previous research assessed a constellation of risk factors associated with antenatal insulin therapy, most of the available studies used earlier diagnostic algorithms (mostly two-step approaches) and therefore contain study populations with different risk profiles as compared to patients with GDM identified by the one-step IADPSG approach. While in accordance to our findings some of these previous studies also indicated a significant association between elevated fasting glucose levels and requirement of pharmacotherapy [4-7], more controversial results are reported for elevated post load OGTT glucose: Wong and Jalaludin found that G120 in addition to G0, BMI at booking, and gestational week at diagnosis was independently associated with insulin therapy [6], whereas in another study, which used a comparable diagnostic approach (two-step screening according to the Australasian Diabetes in Pregnancy Society (ADIPS)), the predictive performance of G120 lost its significance in multivariable regression [4]. A further Australian study identified several clinical determinants of antenatal insulin treatment including G0, G60, and HbA1c [5]. Nevertheless, these variables explained only a small amount of the variance and in accordance with others the authors concluded that antenatal factors including glycemic measures were insufficient to predict the attributable risk for intensified pharmacotherapy $[5,15]$. This is in contrast to our finding in GDM patients identified by the one-step IADPSG approach, where aggregated information of glycemic as well as clinical risk factors showed an acceptable accuracy with an ROC-AUC ranging from 71.1\% (glycemic parameters) to $77.5 \%$ (glycemic parameters and clinical risk factors). Only two further studies were identified using the IADPSG criteria for GDM classification: Bakiner et al. found that G0 (ROC-AUC: 73.4\%) and HbAlc (ROC-AUC: 67.7\%) were independently associated with antenatal insulin treatment in a retrospective study on 300 pregnancies [16]. However, dynamic OGTT glucose values were not reported in this study. Mitra et al. observed a significant association between G60 and antenatal insulin 


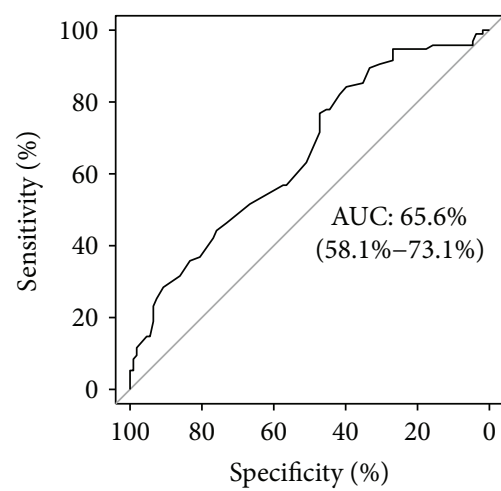

(a)

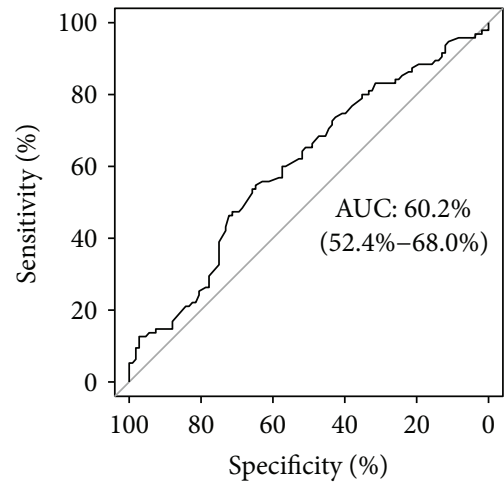

(c)

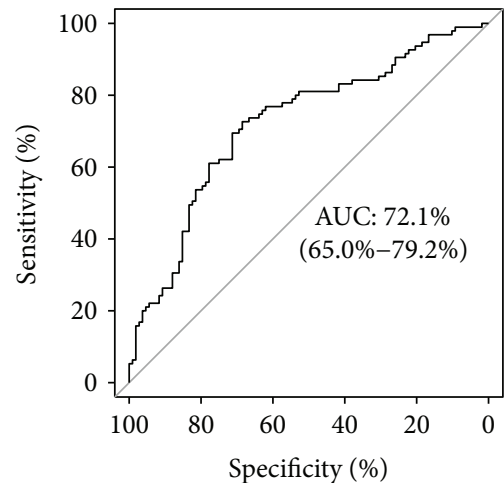

(e)

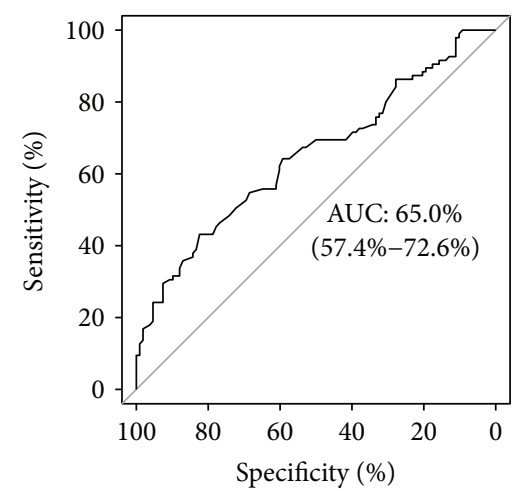

(b)

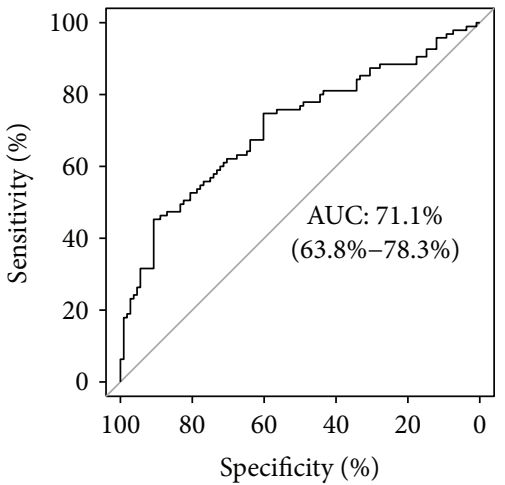

(d)

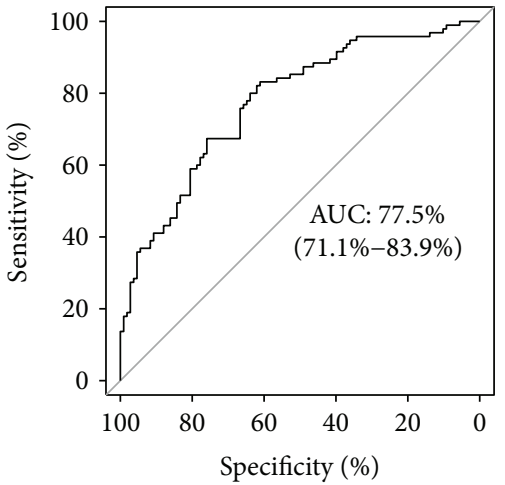

(f)

FIGURE 1: ROC curves for prediction of initiation of pharmacotherapy by OGTT glucose measurements at fasting (a), 60' (b), and $120^{\prime}(\mathrm{c})$ after oral glucose load; combined information of OGTT glucose measurements (d); clinical predictors (age, pregestational BMI, parity, history of GDM, family history with type 2 diabetes, and time of diagnosis) (e); and OGTT glucose measurements and clinical predictors (f).

treatment with an ROC-AUC of $83.1 \%$ [17]. However, the sample size of this study was very small with 8 out of 83 patients requiring pharmacotherapy.

The importance of dynamic glucose measurements for risk stratification is also supported by previous research in nonpregnant patients indicating strong associations between elevated fasting and dynamic OGTT glucose measures with decreased insulin sensitivity and $\beta$-cell dysfunction [18]. Accordingly, a higher degree of insulin resistance and impaired insulin secretion was recently found to characterize patients with GDM requiring pharmacotherapy [19]. A standardized examination of HbAlc at the time of diagnosis was not available in our study, which could be seen as a limitation of this work. However, in contrast to dynamic measures of glucose levels, HbAlc is a weak surrogate of insulin sensitivity or secretion and gives almost no additive information to glycemic parameters assessed during the OGTT [20]. HbAlc is further subjected to pregnancy-specific changes $[21,22]$. Although we provided an exemplary decision tree including the main predictors for intensified therapy identified by our study, this algorithm needs further validation. Of note, we were not able to assess lifestyle habits or several components of the "metabolic syndrome" due to the retrospective nature of this work. This data could be of additional importance and needs to be addressed in future prospective studies. 


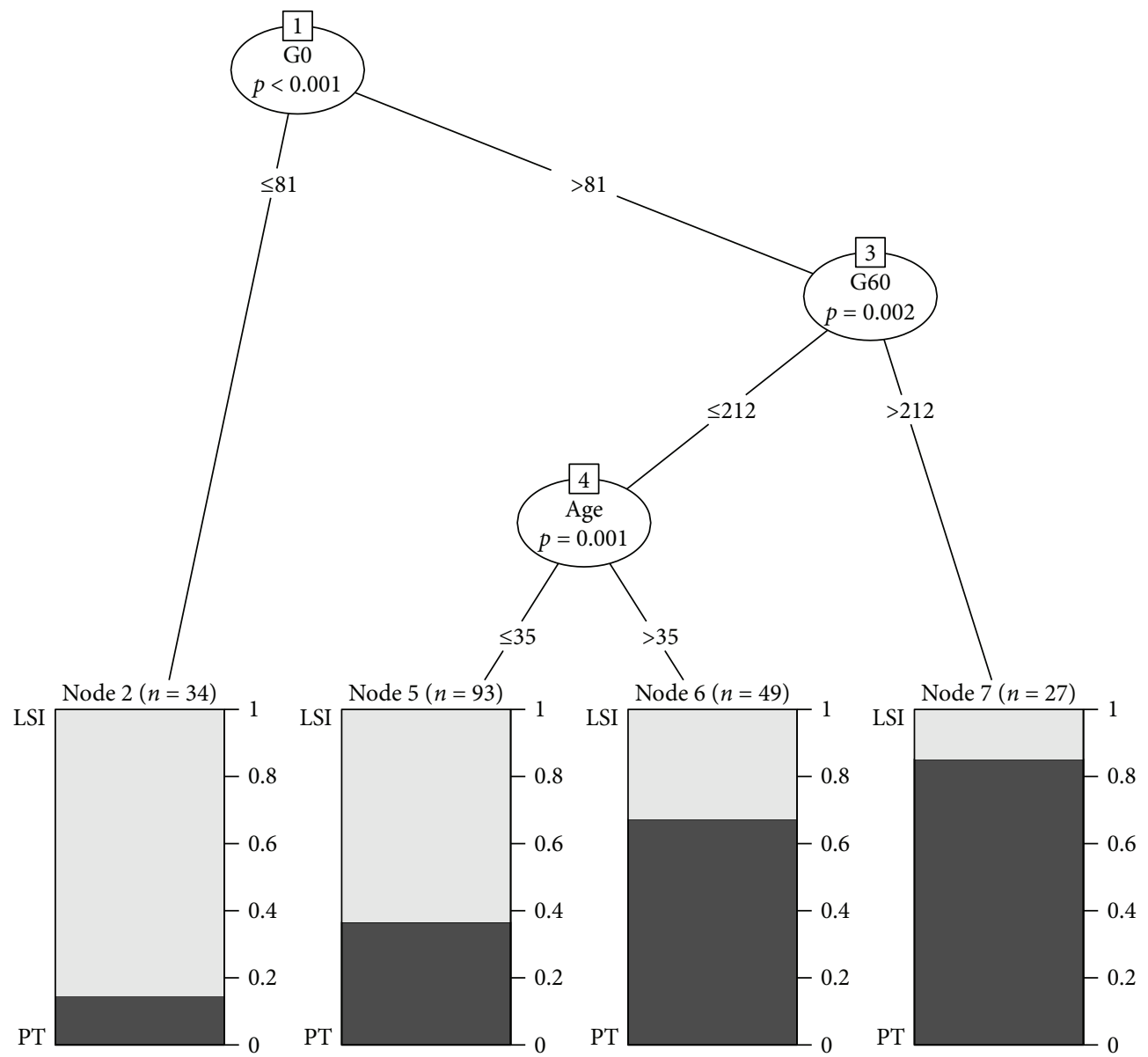

FIGURE 2: Example of a conditional inference tree for the prediction of intensified pharmacologic intervention in GDM patients. Bonferroni adjusted $p$ values are given for each inner node, and the fraction of lifestyle intervention (LSI) and initiation of pharmacotherapy (PT) is provided at each terminal node.

\section{Conclusions}

In summary, we found that fasting and dynamic OGTT glucose measures in addition to clinical risk factors showed promising properties for risk stratification in GDM patients classified by the recently established IADPSG criteria. These findings should be considered in future studies to establish an accurate separation for the early treatment of high-risk patients.

\section{Data Availability}

Data are available from the corresponding author for researchers who meet the criteria for access to confidential data. Please contact Christian Göbl (email: christian. goebl@meduniwien.ac.at).

\section{Conflicts of Interest}

The authors declare that there is no conflict of interest regarding the publication of this paper.

\section{References}

[1] International Association of Diabetes and Pregnancy Study Groups Consensus Panel, "International Association of Diabetes and Pregnancy Study Groups Recommendations on the Diagnosis and Classification of Hyperglycemia in Pregnancy," Diabetes Care, vol. 33, no. 3, pp. 676-682, 2010.

[2] World Health Organisation, Diagnostic Criteria and Classification of Hyperglycaemia First Detected in Pregnancy, WHO Press, World Health Organization, Geneva, Switzerland, 2013.

[3] D. A. Sacks, D. R. Hadden, M. Maresh et al., "Frequency of gestational diabetes mellitus at collaborating centers based on IADPSG Consensus Panel-Recommended Criteria: the Hyperglycemia and Adverse Pregnancy Outcome (HAPO) Study," Diabetes Care, vol. 35, no. 3, pp. 526-528, 2012.

[4] R. A. Barnes, T. Wong, G. P. Ross et al., "A novel validated model for the prediction of insulin therapy initiation and adverse perinatal outcomes in women with gestational diabetes mellitus," Diabetologia, vol. 59, no. 11, pp. 2331-2338, 2016.

[5] T. Pertot, L. Molyneaux, K. Tan, G. P. Ross, D. K. Yue, and J. Wong, "Can common clinical parameters be used to identify patients who will need insulin treatment in gestational diabetes mellitus?," Diabetes Care, vol. 34, no. 10, pp. 2214-2216, 2011. 
[6] V. W. Wong and B. Jalaludin, "Gestational diabetes mellitus: who requires insulin therapy?," Australian and New Zealand Journal of Obstetrics and Gynaecology, vol. 51, no. 5, pp. 432-436, 2011.

[7] V. H. González-Quintero, N. B. Istwan, D. J. Rhea et al., “Antenatal factors predicting subsequent need for insulin treatment in women with gestational diabetes," Journal of Women's Health, vol. 17, no. 7, pp. 1183-1187, 2008.

[8] C. H. Lin, S. F. Wen, Y. H. Wu, and M. J. Huang, "Using the 100-g oral glucose tolerance test to predict fetal and maternal outcomes in women with gestational diabetes mellitus," Chang Gung Medical Journal, vol. 32, no. 3, pp. 283-289, 2009.

[9] HAPO Study Cooperative Research Group, B. E. Metzger, L. P. Lowe et al., "Hyperglycemia and adverse pregnancy outcomes," New England Journal of Medicine, vol. 358, no. 19, pp. 1991-2002, 2008.

[10] American Diabetes Association, "15. Diabetes advocacy:standards of medical care in diabetes-2018," Diabetes Care, vol. 41, Supplement 1, pp. S152-S153, 2017.

[11] T. Hothorn, K. Hornik, and A. Zeileis, "Unbiased recursive partitioning: a conditional inference framework," Journal of Computational and Graphical Statistics, vol. 15, no. 3, pp. 651-674, 2006.

[12] J. P. Fine and R. J. Gray, "A proportional hazards model for the subdistribution of a competing risk," Journal of the American Statistical Association, vol. 94, no. 446, pp. 496509, 1999.

[13] R Core Team, R: A Language and Environment for Statistical Computing, R Foundation for Statistical Computing, Vienna, Austria, 2017.

[14] T. Cundy, E. Ackermann, and E. A. Ryan, "Gestational diabetes: new criteria may triple the prevalence but effect on outcomes is unclear," BMJ, vol. 348, article g1567, 2014.

[15] H. Mendez-Figueroa, J. Daley, V. V. Lopes, and D. R. Coustan, "Predicting the need for medical therapy in patients with mild gestational diabetes," American Journal of Perinatology, vol. 31, no. 2, pp. 105-112, 2014.

[16] O. Bakiner, E. Bozkirli, K. Ozsahin, C. Sariturk, and E. Ertorer, "Risk factors that can predict antenatal insulin need in gestational diabetes," Journal of Clinical Medicine Research, vol. 5, 2013.

[17] S. Mitra, P. K. Nayak, J. Sahoo et al., "Predictors for antenatal insulin requirement in gestational diabetes," Gynecological Endocrinology, vol. 30, no. 8, pp. 565-568, 2014.

[18] M. A. Abdul-Ghani, D. Tripathy, and R. A. DeFronzo, "Contributions of $\beta$-cell dysfunction and insulin resistance to the pathogenesis of impaired glucose tolerance and impaired fasting glucose," Diabetes Care, vol. 29, no. 5, pp. 1130-1139, 2006.

[19] K. Benhalima, K. Robyns, P. van Crombrugge et al., "Differences in pregnancy outcomes and characteristics between insulin- and diet-treated women with gestational diabetes," BMC Pregnancy and Childbirth, vol. 15, no. 1, p. 271, 2015.

[20] C. S. Göbl, L. Bozkurt, R. Yarragudi, A. Tura, G. Pacini, and A. Kautzky-Willer, "Is early postpartum HbAlc an appropriate risk predictor after pregnancy with gestational diabetes mellitus?," Acta Diabetologica, vol. 51, no. 5, pp. 715-722, 2014.
[21] K. Hashimoto, T. Osugi, S. Noguchi et al., "A1C but not serum glycated albumin is elevated because of iron deficiency in late pregnancy in diabetic women," Diabetes Care, vol. 33, no. 3, pp. 509-511, 2010.

[22] R. C. E. Hughes, J. Rowan, and C. M. Florkowski, "Is there a role for HbA1c in pregnancy?," Current Diabetes Reports, vol. 16, no. 1, p. 5, 2016. 


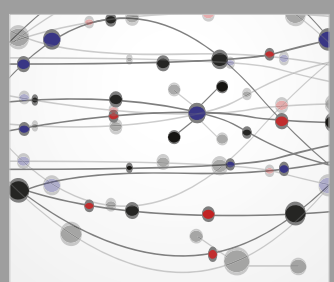

The Scientific World Journal
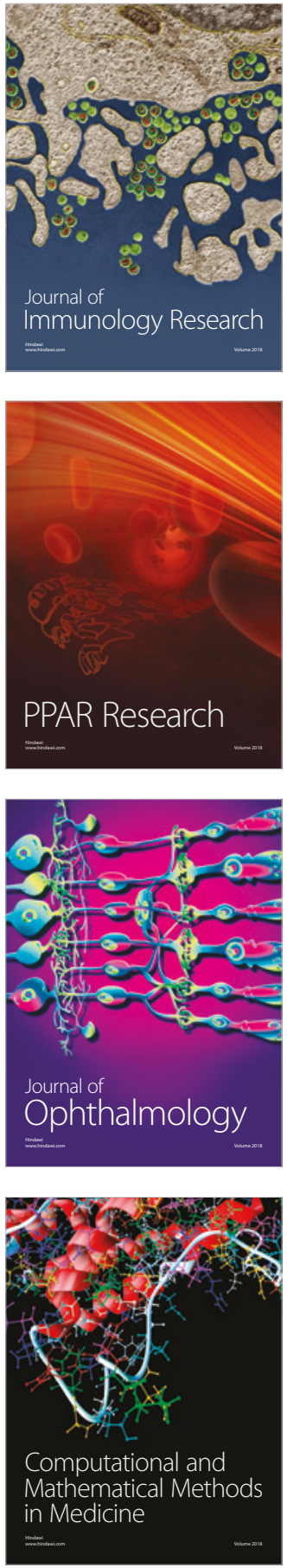

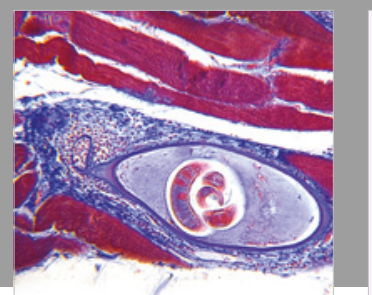

Gastroenterology Research and Practice

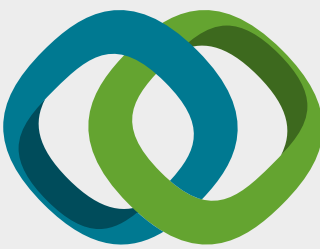

\section{Hindawi}

Submit your manuscripts at

www.hindawi.com
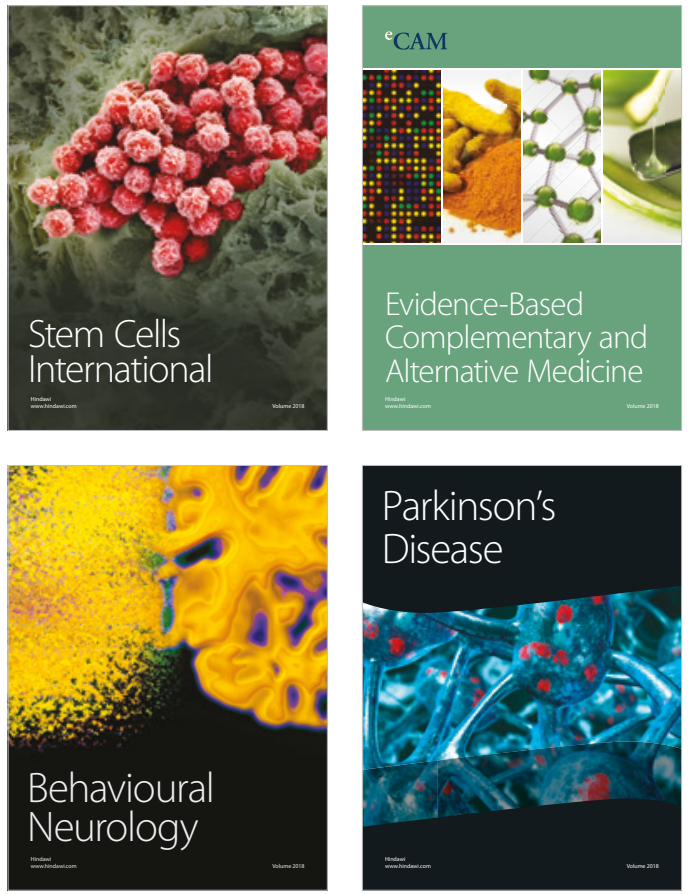

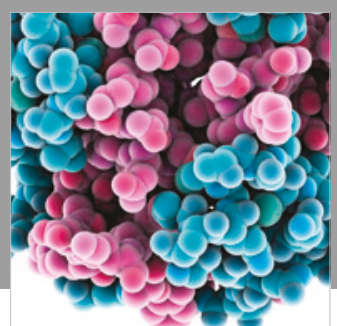

ournal of

Diabetes Research

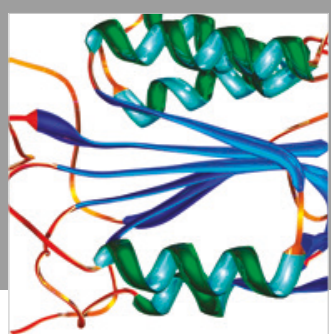

Disease Markers
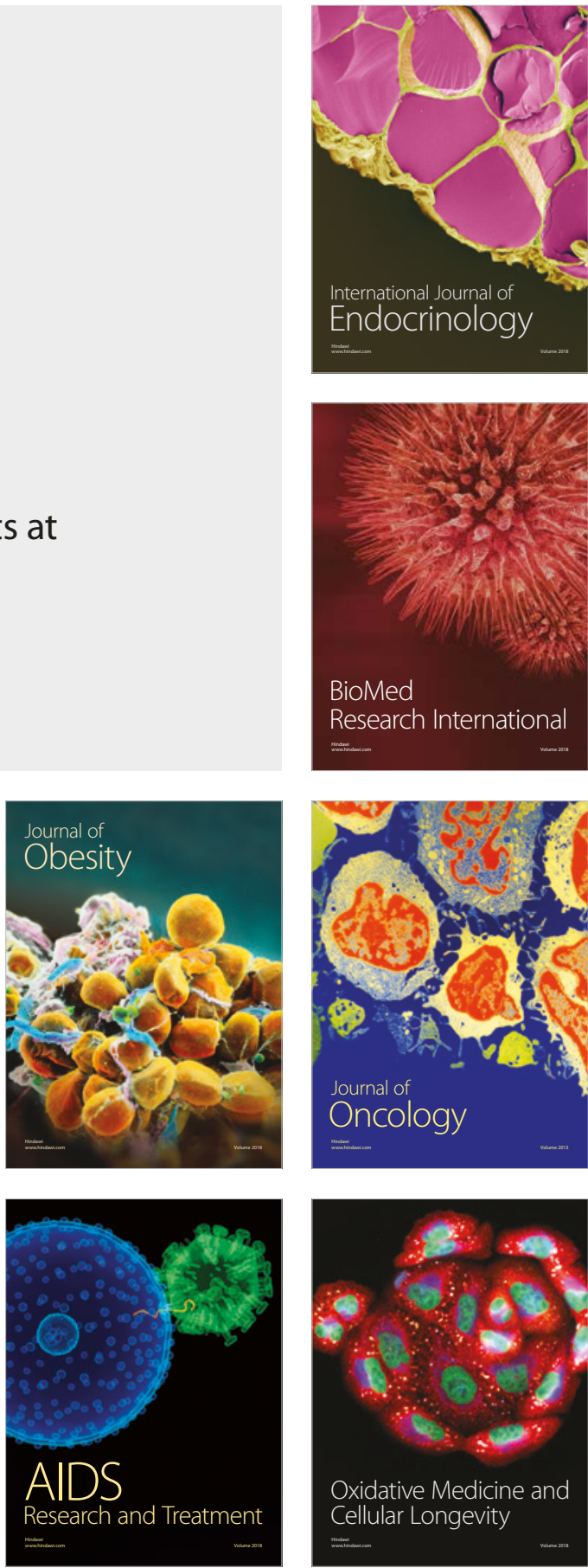